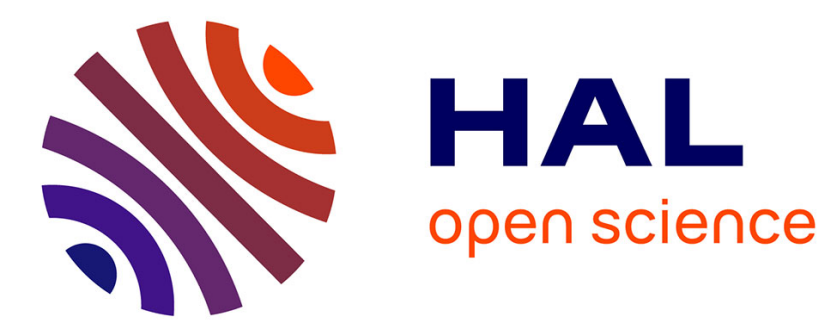

\title{
Use of image treatment for the understanding of the influence of crystal defects in piezoelectric materials
}

\author{
M. Pilard, Y. Epelboin, B. Capelle, J. Detaint, J. Schwartzel, A. Zarka
}

\section{To cite this version:}

M. Pilard, Y. Epelboin, B. Capelle, J. Detaint, J. Schwartzel, et al.. Use of image treatment for the understanding of the influence of crystal defects in piezoelectric materials. Journal de Physique IV Proceedings, 1994, 04 (C2), pp.C2-53-C2-60. 10.1051/jp4:1994207 . jpa-00252475

\section{HAL Id: jpa-00252475 https://hal.science/jpa-00252475}

Submitted on 1 Jan 1994

HAL is a multi-disciplinary open access archive for the deposit and dissemination of scientific research documents, whether they are published or not. The documents may come from teaching and research institutions in France or abroad, or from public or private research centers.
L'archive ouverte pluridisciplinaire HAL, est destinée au dépôt et à la diffusion de documents scientifiques de niveau recherche, publiés ou non, émanant des établissements d'enseignement et de recherche français ou étrangers, des laboratoires publics ou privés. 


\title{
Use of image treatment for the understanding of the influence of crystal defects in piezoelectric materials
}

\author{
M. PILARD, Y. EPELBOIN, B. CAPELLE, J. DETAINT** J. SCHWARTZEL ${ }^{*}$ and A. ZARKA \\ Laboratoire de Minéralogie-Cristallographie, Université P. et M. Curie et D. Diderot, Associé au C.N.R.S., \\ case 115, 75252 Paris cedex 05, France \\ France Telecom CNET, Département PAB/BAG/MCT/CRL, 92220 Bagneux, France
}

\begin{abstract}
: the propagation of surface or bulk acoustic waves in piezoelectric materials has been studied using the time structure of synchrotron $X$-ray beam. A precise state of vibration may be frozen in a stroboscopic topograph. The analysis of such images may be difficult since crystal defects induce highly contrasted areas which mask interesting details. Numerical image treatment give very efficient solutions to extract this "hidden" information. Fourier filtering is one of the methods and has been applied to the topographs, to study the interaction of the defects with the acoustic waves.
\end{abstract}

\section{INTRODUCTION}

Crystal distortions are commonly studied by mean of X-ray topography, since lattice deformations give rise to black and white contrasts in the topograph $(1,2)$, characterizing the crystal defects. Acoustic waves inside or along the surface of a piezoelectric material induce time periodic deformations. It is possible to view the propagating waves, using the time structure of synchrotron radiation. This beam is made of periodic X-ray pulses which allow to "freeze" a given state of deformation during topograph recording. The frequency of the X-ray beam at LURE/DCI is $f_{\text {beam }}=3.169280 \mathrm{MHz}$. One may follow the different states of the acoustic waves by shifting the phase between the beam and the wave( 3 ).

Up to now such stroboscopic topographs were mainly recorded on photographic films, and the analysis is limited to a visual one. It means that a large part of the information is never analysed(4), particularly where strong contrasts due to crystal distortions mask the weak details of interest. In this paper we show that suitable numerical image treatment allows to extract important features from the Laue topographs of surface acoustic wave devices. Some simple treatments may be applied on line during the experiment, other require more time for analysis and computing and are performed after the experiment on topographs which present features of special interest.

\section{TOPOGRAPH DIGITISATION}

We will first describe this operation since the amount of information retrieved from an image strongly depends on this operation. 
2.1. Experimental setting

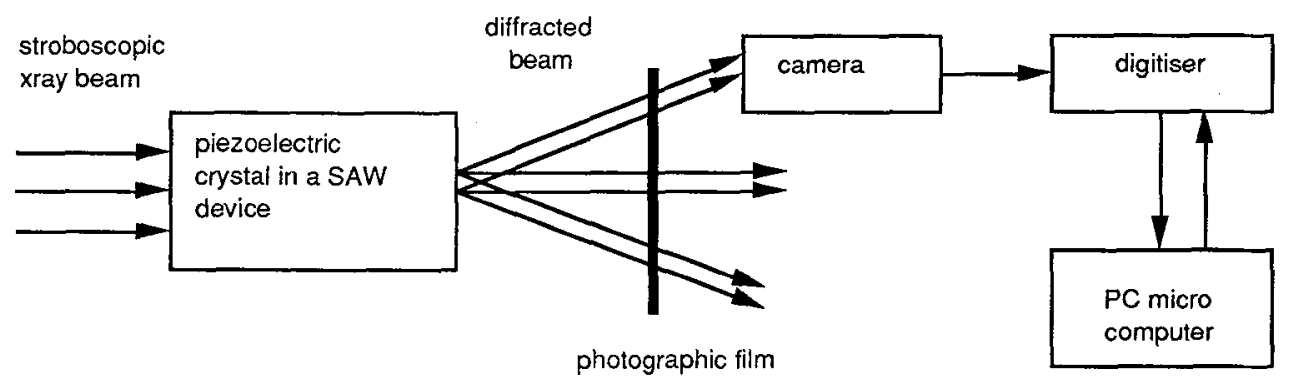

fig. 1: experimental setup for topograph

digitisation

Laue topographs are digitised by means of a high resolution TV camera (Sofretec CV154NV) (fig. 1). First a photographic Laue image is recorded where many Laue spots are visible. This allows to choose the one presenting the best contrast to align the camera. The field of view is sufficient to view one Laue pattern. The resolution of the output image is 25 microns about. It is limited by the Xray converter(5), a fluorescent screen. The efficiency of such a converter (ideally each incident $X$ photon should be detected and converted in "visible" photons) is directly linked to the plate thickness and the size of the grain: larger the grain size, higher is the efficiency, but worse is the resolution. Up to now the best cameras provide a 15 to $20 \mu$ m resolution.

The video signal from the camera is sent to a Matrox IP8 digitizer card, plugged in a PC microcomputer. The image is sampled as a $512 \times 512$ pixels picture. Each pixels takes a value between 0 and 255, representing the diffracted intensity in the corresponding area of the image. It would be useless to sample the image with a higher resolution since it is already limited by the camera itself. Over sampling adds no information(6), and increases the amount of data to be processed without any use.

Pixel values in the digitised image could be encoded on more than eight bits to achieve a better dynamics for the intensities. This is useful for camera with a high signal to noise ratio only, as it will be explained in the next paragraph.

\subsection{Multiple video frames acquisition}

The acquisition time corresponds to one videoframe, i.e. $1 / 25$ th second. This is not sufficient to obtain a satisfactory signal to noise ratio. Furthermore the electronic noise of the whole system reduces dramatically the signal to noise ratio.

Adding many video frames together allows to collect more photons per pixel and to enhance the signal to noise ratio. Since the quantum and electronic noises have a poissonian statistical distribution(7), the signal to noise ratio thus the number of significant grey levels increases as the square root of the number of accumulated video frames. For instance, a camera which can collect 500 photons per pixel during a single videoframe $(1 / 25 \mathrm{th} \mathrm{sec})$ gives $500 /(2 \sqrt{500})=11$ significant values for the measured intensities. Summing 64 video frames, the number of significant values becomes $(64 \times 500) /(2 \times 8 \times \sqrt{500})=88$. The same explanation is valid for photographic films and it explains why the exposure time must be large enough to obtain a good contrast. However one is limited by saturation effects. 


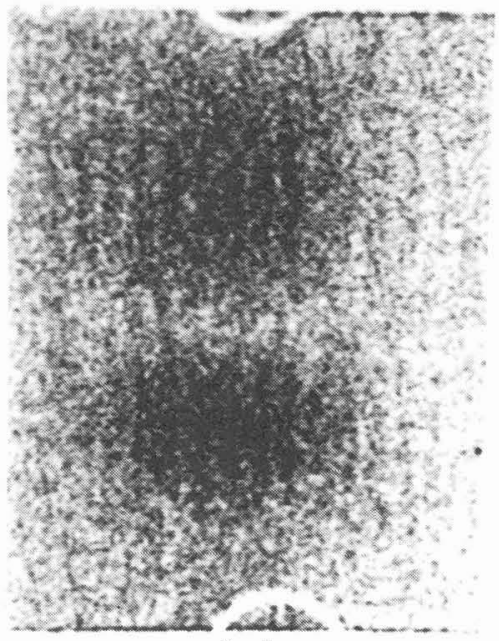

fig.2.a.

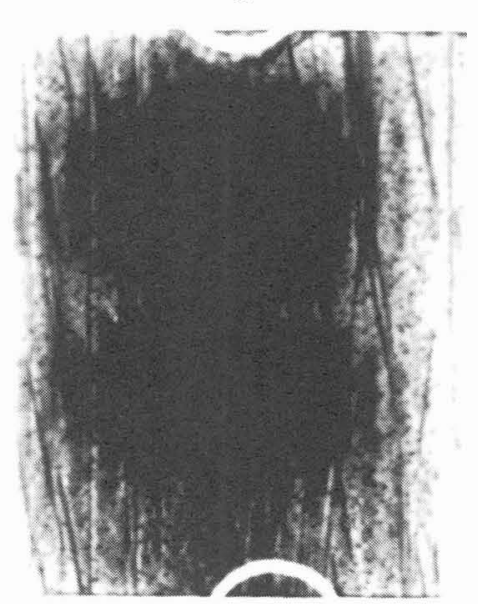

fig.2.c.

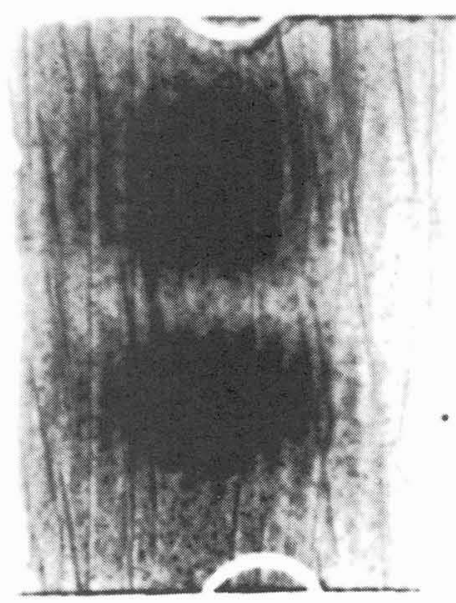

fig. 2.b. 
The comparison between a single video frame (fig. $2 . a$ ) and the summation of 64 video frames (fig.2.b) shows that noise averaging greatly improves the quality of digitized topographs.

\subsection{Comparison with photographic emulsions}

Photographic films recording and digital recording have their own advantages since they provide complementary informations. The resolution is better in a photographic film ( of the order of $2-3 \mu \mathrm{m}$ for the best ones), and many Laue pattern are simultaneously recorded. But the time of exposure plus the processing time, of the order of several minutes, is constraining for the study of dynamical phenomenon. In such case topograph is preferable. Acquisition time, from less than $1 \mathrm{~s}$ up to 40 seconds for 32 accumulated video frames, provides a better time resolution. Digitised images can be immediately displayed; the gain in time is not negligible when the experiment time is limited. Moreover since our software allows on line adjustment of digitisation parameters such as the contrast, one may easily fit the dynamics of grey levels to the range of interesting intensities for each topograph, and avoid saturation effects as observed in photographic films.

One must also emphasise that photographic films allow a qualitative analysis only. Since a digitised topograph is a set of numerical data, mathematical treatments may be applied, providing new quantitative information. Some of these treatments are performed on line, during the experiment, and other, requiring more time for analysis and computing, are processed on more powerful computers after the experiment.

\section{EXPERIMENTAL RESULTS}

The interaction between crystal defects and surface acoustic waves ( SAW ) is an important feature since it is linked to SAW devices quality. This coupling is expected from simulations $(8,9)$ but has never been observed experimentally.

Fig. $3 a$ and fig. $3 b$ present digitised topographs of a quartz crystal in a SAW device. In fig.3a the quartz crystal is not under excitation: only crystal defects, mainly dislocations, are visible in the topograph. Fig. $3 \mathrm{~b}$ presents the same topograph, and the crystal is excited through its transducers not visible on the image. A contrasts due to SAW is visible as vertical fringes, overlapping with crystal dislocations, and a precise analysis is impossible.

The visibility of the vibrating mode is greatly improved by a numerical comparison of the two topographs. This is made by a simple images subtraction, since both images are perfectly superimposable. Fig.3c is the result of the subtraction of the topographs.

It becomes obvious that the propagation of surface acoustic waves is disturbed in the area with a high density of dislocations. The vibration takes its maximum where the crystal is rather perfect.

Further investigations will be needed for a complete understanding of this interaction; this example of numerical treatment shows that digitised topographs provides essential information for this study.

The subtraction technique has been applied to various crystals in S.A.W. devices, showing undoubtedly a coupling between surface acoustic waves and dislocations: the strain field around these defects appear to be modified by the acoustic wave. Results will be presented in a future paper.

\section{OFF LINE ANALYSIS}

In this paragraph we present more powerful methods, to extract more informations, either from photographic films or from digitised topographs. Since more time is needed to find the adequate treatment and its parameters this is restricted to post-experiment processing.It also requires a more powerful computer.

We use Fourier filtering in an interactive software, called TRN, now under development. Other methods, such as wavelet decomposition or entropy maximisation, are under study. 


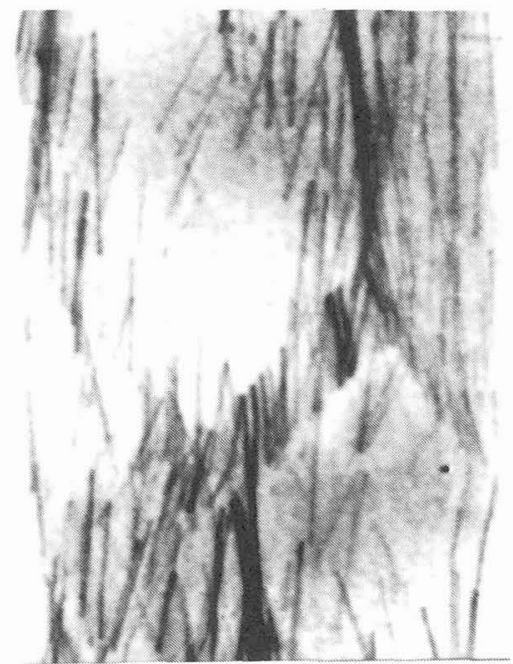

fig.3.a.

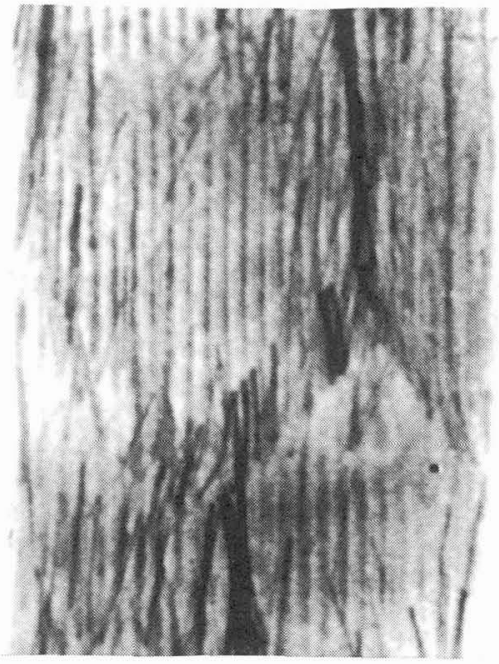

fig.3.b.

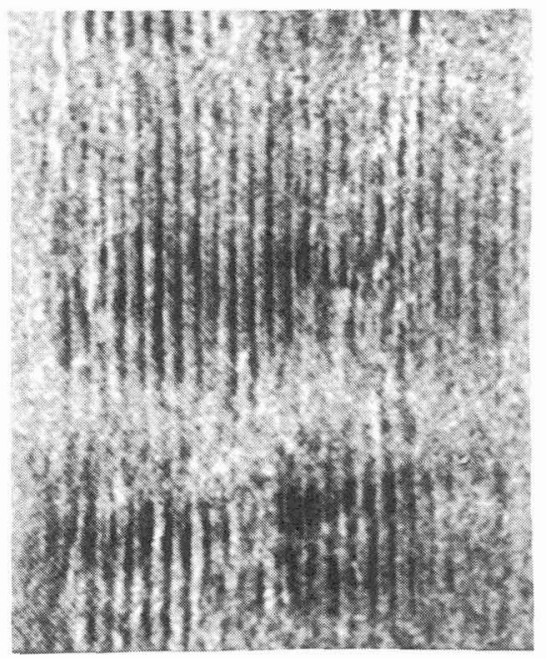

fig.3.c.

fig. (3): Laue topographs of a quartz resonator. Vertical dimension $10 \mathrm{~mm}$ about. 32 accumulated frames.

(a.) crystal without excitation: only crystal defects are visible. Notice a high density of dislocations in the center of the topograph.

(b.) Stroboscopic image of the same crystal under excitation: surface acoustic waves appear as vertical fringes intricated with crystal defects.

(c.) Computed difference between (a) and (b): the noise being additive, the difference lessens the signal to noise ratio. 
Discrete Fourier transform ( DTF ) is the only coherent way to perform frequential decomposition of a sampled signal. DFT is computed using the Cooley algorithm(10), minimising the processing time. Once the Fourier transform of an image has been processed, frequential filters may be applied for various purposes: image restoration, enhancement of features of particular interest. The greatest care must be taken to keep the mathematical consistency of the filtering process.

\subsection{Fourier filtering}

A digitised image is a set of real values representing intensities. Thus its Fourier transform is centrosymmetric. To keep these properties one must apply only centrosymmetric frequential filters otherwise the result of the filtering is an image with complex intensities!

A frequential filter may be defined in Fourier space as a function taking the value 1 where the frequential components must be retained, and the value 0 where the components have to be suppressed. Such filters introduce artefacts which appear as diffraction fringes around some features in direct space. These artefacts are due the abrupt edges of the frequential filter. Hence filter edges must be smoothed. Many models of transitions have been introduced to minimise such effects. We use the "optimum filter" defined by Tournarie(11) which induces a minimum of artefacts. TRN allows to build various classes of filters suitable for specific purposes. One of them is isotrope filtering, using circular filters centred at the origin of Fourier space: all the directions of the direct image are processed with the same filtering. Elliptical and polar filters are used to enhance or remove features of particular orientation. A combination of these different filterings is often necessary to achieve a precise and efficient treatment, as illustrated with the following example.

\subsection{Application: wave propagation in a S.A.W. filter}

Fig.4a has been digitised from the photographic image of a Lithium Niobate S.A.W. filter. The principle of such filters relies on piezoelectric properties of the material: the input inter digital transducer (IDT) at the low right side of the image, converts the input electrical signal in a acoustic signal. The induced surface acoustic wave is then guided through a multistrip coupler (MSC). At the upper left side, an output IDT converts the acoustic signal in a electric one. The quality of the filter depends, among other parameters, on the interaction between the surface acoustic waves and crystal defects, and on energy dissipation in the crystal.

The filter in fig.4a was under excitation during the film recording. The surface acoustic waves are not clearly visible under the MSC and the IDTs since strong black contrasts, due to strain field in the crystal, mask completely the corresponding fringes. Waves are only visible along the edges of the MSC.

Fig.4c is the same topograph after suitable Fourier filtering. First, low frequencies in the Fourier transform ( up to $5 \%$ of the maximum visible frequencies, fig. $4 \mathrm{~b}$ ) have been suppressed using a circular high pass filter. It removes the slow varying black contrast and thus reveals the weak details under the MSC. Moreover, the analysis of the Fourier transform (fig. $4 b$ ) shows clearly a cluster of spatial frequencies corresponding to the shape of the propagating wave. Weighing these frequencies with a polar filter enhances wave visibility.

It is now possible in fig. $4 \mathrm{c}$ to look at the surface acoustic wave all along the IDTs and MSC. Fringes are visible all around them: acoustic energy dissipation takes place despite the presence of an acoustic absorbent placed around the MSC. The emitter transducer, bottom part of fig.4a and fig.4c, shows an intricate radiation diagram with several lobes at its left end. This creates distortions in the crystal and disturbs the shape of the surface acoustic wave.

Fig. 4 is a demonstrative example pointing out that an image contains more information than it is visible: the cluster of high frequencies in fig. 4 .b shows clearly that information about the surface acoustic waves is present in the original topograph but the eye cannot catch it: one of the aims of image processing is to reveal it. 


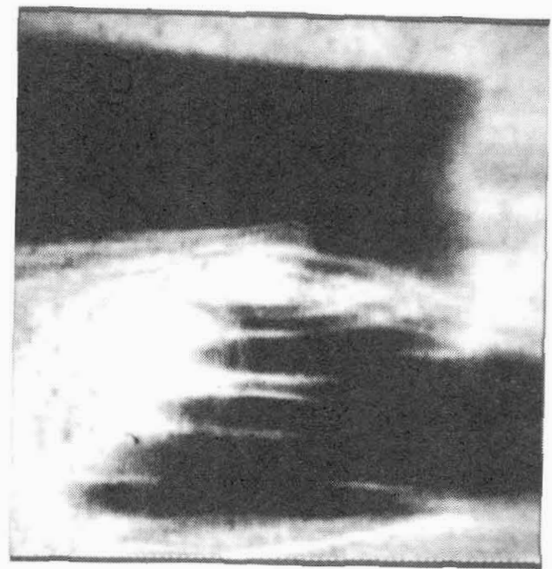

fig.4.a.

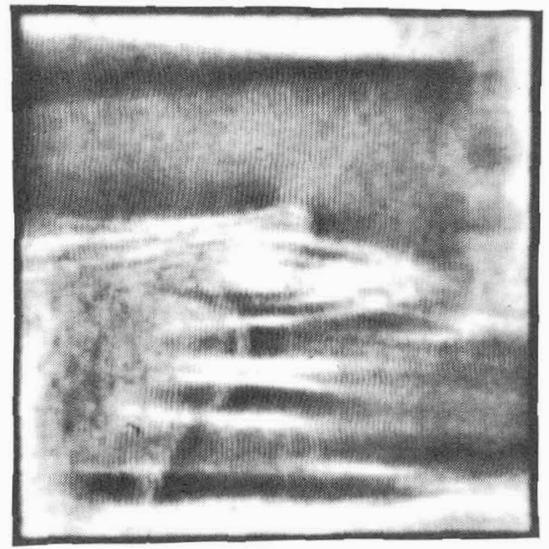

fig.4.c.

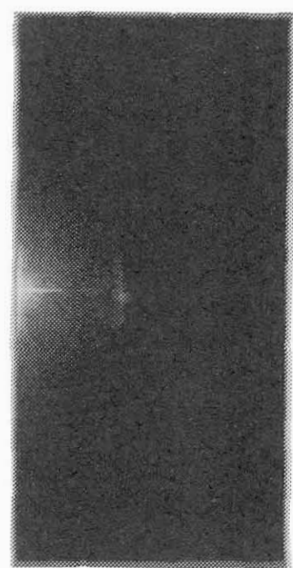

fig.4.b.

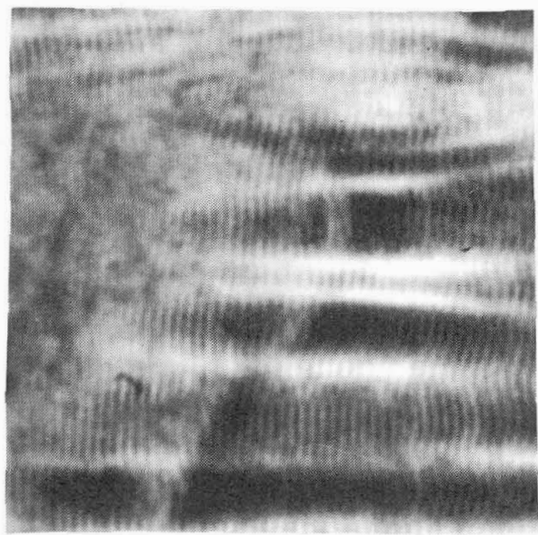

fig. (4): Stroboscopic topography of a Lithium Niobate crystal in a S.A.W. device; the crystal is under excitation. Vertical dimension $10 \mathrm{~mm}$ about. The image was first recorded on film during the experiment, and then digitized for numerical processing.

(a) Original topograph; the black area corresponds to the waveguide. This strong contrast, due to crystal distortions, fully masks the surface acoustic wave contrast.

(b) Fourier transform of topograph (a); half space only has been represented since it is centrosymmetric. The origin is at the middle of the left edge; the peak at the origin corresponds to the sum of all the intensities of (a). A cluster of high Fourier components is clearly visible in the center of the Fourier image; it corresponds to S.A.W. in direct space.

(c) Topograph after Fourier filtering. Low frequencies have been suppressed, while the cluster of high frequencies has been enhanced: the propagating waves are clearly visible. 


\section{CONCLUSION}

The previous results show that numerical image treatments provide useful tools to study the propagation of surface acoustic waves in piezoelectric materials. It is possible to study how defects interact with the propagating waves. Furthermore, image processing allows to enhance the visibility of energy dissipations which can be precisely located as seen in the S.A.W. filter.

Finally, X-rays stroboscopic topography is a powerful tool for the study of saw and bulk acoustic waves devices, deserving a deeper analysis than just a visual one. Human eye has not the capability to grab all the information contained in a topograph; numerical image processing helps to a richer analysis. We have applied mainly Fourier filtering, but other methods such as wavelet decomposition or entropy maximisation, providing complementary tools for topographs analysis, may be of great interest in the future.

\section{References:}

(1) S. Tagaki, Acta Cryst., 23 (1962), 23

(2) N. Kato , J. Phys. Soc. Jpn, 18 (1963), 775

(3) A. Zarka, B. Capelle, J. Detaint \& J. Schwartzel, J. Appl. Cryst. , 21 (1988), 967

(4) Y. Epelboin, F. Morris \& A. Rimsky, J. Phys. D, (1992)

(5) B.K. Tanner \& J.K. Cringean, Prog. Crystal Growth and Charact., 14 (1987), 403-424

(6) C.E. Shannon, Bell Systems Tech. J., 27 (1948), 327, 623

(7) P.R. Buseck,Y. Epelboin\&A. Rimsky, Acta Cryst., A44 (1988), 975-986

(8) Y. Zheng, A. Zarka, B. Capelle, J. Detaint \& J. Schwartzel, Acta Cryst. A45 (1989), 275-285

(9) Y. Epelboin \& J.R. Patel, J. Appl. Phys., 53 (1982), 271

(10) J.W. Cooley \& J.W.Tuckey, Math. Comput. 19 (1965) 297

(11) M.Tournarie, Bull. Soc. Franç. Miner. Cris. 81 (1958) 278 